[1] R. O. Davies, Separate approximate continuity implies measurability, Proc. Cambr. Phil. Soc. 73 (1973), pp. $461-465$.

[2] Z Grande, Sur la mesurabilité des fonctions de deux variables, Bull. Acad. Polon. Sci. Sér. Sci. Math. Astronom. Phys. 21 (1973), pp. 813-816.

[3] - On measurability of functions of two variables, Proc. Cambr. Phil. Soc. (in press).

[4] S. Saks, Theory of the Integral, Warszawa 1937.

Accepté par la Rédaction le 21, 10.1974

\section{On bouquets}

by

Anna Gmurczyk (Warszawa)

Abstract. The paper concerns with bouquets of metric continua, in particular with spherical bouquets and usual spherical bouquets. Some results are the following:

Every bouquet of ANR's is movable.

A bouquet is FAR if and only if all its leaves are FAR's.

A bouquet of ANR's is homeomorphic to the inverse limit of a sequence of finite subbouquets with bonding maps being retractions.

Every spherical bouquet is of the same shape as a locally connected spherical bouquet.

For usual $n$-dimensional spherical bouquets $(n>1)$ two fundamental sequences are homotopic whenever they are homologic.

The classification of spaces, in particular of compact spaces, into classes called shapes is based only on global properties of those spaces, and thus it is far less precise than topological classification. The question arises how to find the singles possible space in each class. E.g., for the class of each plane continuum there exists a representative which is a finite or countable bouquet of 1 -spheres, i.e., a set homeomorphic to one of the subsets

and

$$
\begin{aligned}
X_{k} & =\bigcup_{i=1}^{k}\left\{(x, y) \in E^{2}:\left(x-\frac{1}{i}\right)^{2}+y^{2}=\left(\frac{1}{i}\right)^{2}\right\} \text { for } k=1,2, \ldots \\
X & =\bigcup_{i=1}^{\infty}\left\{(y, x) \in E^{2}:\left(x-\frac{1}{i}\right)^{2}+y^{2}=\left(\frac{1}{i}\right)^{2}\right\}
\end{aligned}
$$

of the plane $E^{2}$.

The aim of this paper is to study the properties of bouquets in more general sense.

1. Basic definitions. In [1]-[5] K. Borsuk introduced the basic notions of shape. We recall some of the basic definitions.

Let $X$ and $Y$ be two compacta lying in an AR(M)-space $M$.

A sequence of continuous maps $f_{k}: M \rightarrow M$ is said to be a fundamental sequence from $X$ to $Y$ (notation: $f=\left\{f_{k}, X, Y\right\}$ ) if for every neighborhood $V$ of $Y$ there is a neighborhood $U$ of $X$ such that

$\left.\left.f_{k}\right|_{v} \simeq f_{k+1}\right|_{v} \quad$ in $V \quad$ for almost all $k$. 
Two fundamental sequences $f=\left\{f_{k}, X, Y\right\}$ and $g=\left\{g_{k}, X, Y\right\}$ are said to be homotopic (notation: $f \simeq \underline{g}$ ) if for every neighborhood $V$ of $Y$ there is a neighborhood $U$ of $X$ such that

$\left.\left.f_{k}\right|_{U} \simeq g_{k}\right|_{U} \quad$ in $V \quad$ for almost all $k$.

By the fundamental identity sequence from $X$ to $X$ we understand a fundamental sequence id $_{X}=\left\{i_{k}, X, X\right\}$ such that $i_{k}(x)=x$ for every $x \in X$ and $k=1,2, \ldots$

By the composition $g f$ of two fundamental sequences $\underline{f}=\left\{f_{k}, X, Y\right\}$ and $g=\left\{g_{k}, Y, Z\right\}$ we understand the fundamental sequence $\left\{g_{k} f_{k}, X, Z\right\}$.

If there exist two fundamental sequences $f=\left\{f_{k}, X, Y\right\}$ and $g=\left\{g_{k}, Y, X\right\}$ such that $g f \simeq \operatorname{id}_{X}$ and $f g \simeq \operatorname{id}_{Y}$, then we say that $X$ and $Y$ have the same shape (notation: $\overline{S h} \bar{X}=\operatorname{Sh} Y$ ).

Replacing in those definitions the compacta $X, Y$ by the pointed compacta $\left(X, x_{0}\right) \subset\left(M, x_{0}\right),\left(Y, y_{0}\right) \subset\left(M, y_{0}\right)$ and also the neighborhoods $U, V$ by the pointed neighborhoods $\left(U, x_{0}\right),\left(V, y_{0}\right)$ we get the notions of the fundamental pointed sequence $f=\left\{f_{k},\left(X, x_{0}\right),\left(Y, y_{0}\right)\right\}$, of the homotopy of pointed fundamental sequences and of the shape of pointed compacta.

A closed subset $A$ of a compact set $X \subset M$ is said to be a fundamental retract of $X$ if there exists a fundamental retraction of $X$ to $A$, i.e., a fundamental sequence $r=\left\{r_{k}, X, A\right\}$ satisfying the condition $r_{k}(x)=x$ for every point $x \in A$ and $\bar{k}=1,2, \ldots$

The fundamental retracts of AR-sets are said to be fundamental absolute retracts (FAR-sets) and fundamental retracts of ANR-sets are said to be fundamental absolute neighborhood retracts (FANR-sets).

All the spaces considered in this paper are metric and separable.

The symbols $\varrho, K(x, \varepsilon), K(A, \varepsilon)$ always denote the metric, the open ball with the centre $x$ and the radius $\varepsilon$ and the set of such points $y$ that inf $\varrho(y, a)<\varepsilon$, respectively.

2. The notion of a bouquet. The aim of this part is to give the definition of a bouquet and to prove lemmas needed for studying the properties of bouquets.

(2.1) Defintion. A pointed continuum $(X, a)$ is said to be a bouquet if there exists a family $\mathscr{X}=\left\{X_{\lambda}\right\}_{\lambda \in A}$ of subcontinua of the space $X$ such that the following conditions are satisfied:

(1) $X=\bigcup_{\lambda \in A} X_{\lambda}$

(2) for every $\lambda \in \Lambda$ the set $X_{\lambda}-(a)$ is a component of $X-(a)$,

(3) if $\lambda, \mu \in \Lambda$ are different indexes, then there exist continua $C_{\lambda}, C_{\mu}$ such that $X_{\lambda} \subset C_{\lambda}, X_{\mu} \subset C_{\mu}, X=C_{\lambda} \cup C_{\mu}$, and $C_{\lambda} \cap C_{\mu}=(a)$.

The elements of the family $\mathscr{X}$ are called the leaves and the point $a$ the centre of the bouquet $(X, a)$.

(2.2) Definition. Let $(X, a)$ be a bouquet, $\mathscr{X}=\left\{X_{\lambda}\right\}_{\lambda \in \Lambda}$ the family of its leaves and $(Y, a)$ a pointed continuum such that $Y \subset X$. Then $(Y, a)$ is said to be a subbouquet of $(X, a)$ if it is a bouquet and the family of leaves of $(Y, a)$ is contained in $\mathscr{X}$.

It is easy to see that the intersection and the sum of an arbitrary finite family of subbouquets of $(X, a)$ are also subbouquets of $(X, a)$.

(2.3) LEMMA. If $(X, a)$ is a bouquet and $X=\left\{X_{\lambda}\right\}_{\lambda \in \Lambda}$ is the family of its leaves, then for every $\lambda \in \Lambda$ and for every neighborhood $U$ of $X_{\lambda}$ in $X$ there is a subbouquet $(Y, a)$ of $(X, a)$ such that the set $(X-Y) \cup(a)$ is compact and $X_{\lambda} \subset Y \subset U$.

Proof. Let $\lambda \in \Lambda$ be a fixed index and $U$ a neighborhood of $X_{\lambda}$ in $X$. For every $\mu \in \Lambda, \mu \neq \lambda$, let us denote by $\left(B_{\mu}, a\right)$ and $\left(C_{\mu}, a\right)$ subbouquets of $(X, a)$ such that $X_{\mu} \subset B_{\mu}, X_{\lambda} \subset C_{\mu}, X=B_{\mu} \cup C_{\mu}$ und $C_{\mu} \cap B_{\mu}=(a)$.

Since $X_{\lambda}=\bigcap C_{\mu}$, there is a sequence $\mu_{1}, \mu_{2}, \ldots$ of indexes different from $\lambda$ $\underset{\substack{\mu \in \lambda \\ \mu \neq \lambda}}{\mu \neq \lambda}$

such that

$$
X_{\lambda}=\bigcap_{i=1}^{\infty} C_{\mu_{i}}
$$

Since $X_{\lambda}$ is a compact set, there is a natural number $n_{0}$ such that $\bigcap_{i=1}^{n_{0}} C_{\mu_{i}} \subset U$. The set $Y=\bigcap_{i=1}^{n_{0}} C_{\mu_{l}}$ satisfies the required conditions.

It follows directly from Lemma (2.3) that for every leaf $X_{\lambda}$ of a bouquet $(X, a)$ the set $X_{\lambda}-(a)$ is a quasi-component of $X-(a)$.

(2.4) Lemma. Let $(X, a)$ be a bouquet and $\mathscr{X}=\left\{X_{\lambda}\right\}_{\lambda \in A}$ the family of its leaves. If $\mathscr{U}=\left\{U_{\lambda}\right\}_{\lambda \in A}$ is a family of open subsets of the space $X$ such that $X_{\lambda} \subset U_{\lambda}$ for every $\lambda \in \Lambda$, then there exist finite sequences $\lambda_{1}, \ldots, \lambda_{k}$ of indexes and $\left\{\left(B_{i}, a\right)\right\}_{i=1}^{k}$ of subbouquets of $(X, a)$ such that the following conditions are satisfied:

(1) $X_{\lambda_{j}} \subset B_{j} \subset U_{\lambda_{j}}$ for $j=1, \ldots, k$,

(2) $X=\bigcup_{j=1}^{k} B_{j}$,

(3) $B_{i} \cap B_{j}=(a)$ for $i \neq j, i, j=1, \ldots, k$.

Proof. We can find, by Lemma (2.3), a family $\left\{\left(Y_{\lambda}, a\right)\right\}_{\lambda \in A}$ of subbouquets of $(X, a)$ such that for every $\lambda \in \Lambda$ the set $\left(X-Y_{\lambda}\right) \cup(a)$ is compact and $X_{\lambda} \subset Y_{\lambda} \subset U_{\lambda}$.

$\mathrm{It}$ is clear that there is a sequence $\mu_{1}, \mu_{2}, \ldots$ of elements of $A$ such that $X$ $=\bigcup_{i=1}^{\infty} Y_{\mu l}$

We define the sets $A_{\mu_{1}}, A_{\mu_{2}}, \ldots$ by the formulas

and

$$
A_{\mu_{1}}=Y_{\mu_{1}}
$$

Let us observe that

$$
A_{\mu_{t}}=\left(Y_{\mu_{i}}-\bigcup_{j=1}^{i-1} Y_{\mu_{j}}\right) \cup(a) \quad \text { for } \quad i=2,3, \ldots
$$

$$
\bigcup_{i=1}^{k} A_{\mu_{i}}=\bigcup_{i=1}^{k} Y_{\mu_{i}} \text { for every } k \text { and } \quad X=\bigcup_{i=1}^{\infty} A_{\mu_{i}}
$$


For every $i$ the pointed continuum $\left(A_{\mu_{i}}, a\right)$ is a subbouquet of $(X, a)$, the set $A_{\mu_{i}}-(a)$ is both closed and open in $X-(a)$ and $\left(A_{\mu_{i}}-(a)\right) \cap\left(A_{\mu_{j}}-(a)\right)=\varnothing$ for $i \neq j$.

Let $\varepsilon$ be a positive number such that $A_{\mu_{1}} \not \subset K(a, \varepsilon) \subset U_{\mu_{1}}$.

Since $X=K(a, \varepsilon) \cup \bigcup_{i=1}^{\infty}\left(A_{\mu_{i}}-(a)\right)$ and $X$ is compact, we infer that $A_{\mu_{j}}$ $\subset K(a, \varepsilon)$ for almost all $j$. Let $1=n_{1}<\ldots<n_{k}$ be a sequence of natural numbers such that $A_{\mu_{i}} \not K K(a, \varepsilon)$ for $i=1, \ldots, k$ and $A_{\mu_{j}} \subset K(a, \varepsilon)$ for $j \neq n_{1}, \ldots, n_{k}$.

Let us write $\lambda_{i}=\mu_{n_{i}}$ for $i=1, \ldots, k$. It is clear that $X_{\lambda_{j}} \subset \bigcup_{i=1}^{j} A_{\lambda_{i}}$ for every $1 \leqslant j \leqslant k$.

Before we define the bouquets $\left(B_{i}, a\right)$ for $i=1, \ldots, k$, we shall construct inductively an auxiliary finite sequence $\left(D_{l}^{j-1}, a\right), j=2, \ldots, k, l=1, \ldots, j$, of subbouquets of $(X, a)$.

If $X_{\lambda_{2}} \subset A_{\lambda_{2}}$, then we put $D_{1}^{1}=A_{\lambda_{1}}$ and $D_{2}^{1}=A_{\lambda_{2}}$.

If $X_{\lambda_{2}} \not A_{\lambda_{2}}$, then $X_{\lambda_{2}} \subset A_{\lambda_{1}}$ and we infer by (2.3) that there is a subbouquet $\left(F_{2}, a\right)$ of the bouquet $\left(A_{\lambda_{1}} \cap Y_{\lambda_{2}}, a\right)$ such that $X_{\lambda_{2}} \subset F_{2}, X_{\lambda_{1}} \not \subset F_{2}$ and the set $\left(A_{\lambda_{1}} \cap Y_{\lambda_{2}}-F_{2}\right) \cup(a)$ is compact.

We define $D_{1}^{1}=\left(A_{\lambda_{1}}-F_{2}\right) \cup(a)$ and $D_{2}^{1}=A_{\lambda_{2}} \cup F_{2}$.

Let us suppose that for every $2 \leqslant n \leqslant k$ we have defined a sequence $\left(D_{1}^{j-1}, a\right), \ldots$ $\ldots,\left(D_{j}^{j-1}, a\right), j=2, \ldots, n$, of subbouquets of $(X, a)$ such that the following conditions are satisfied:

(a) $)_{n}$ if $2 \leqslant j \leqslant n$ and $1 \leqslant i \leqslant j$, then $X_{\lambda_{i}} \subset D_{i}^{j-1} \subset U_{\lambda_{i}}$,

(b) $n$ if $2 \leqslant j \leqslant n, 1 \leqslant i, l \leqslant j$ and $i \neq l$, then $D_{i}^{j-1} \cap \dot{D}_{l}^{j-1} \doteq(a)$;

(c) ${ }_{n}$ for every $2 \leqslant j \leqslant n$ and $1 \leqslant i \leqslant j$ the set $D_{l}^{j-1}-(a)$ is both closed and open in $X-(a)$;

(d) $\bigcup_{i=1}^{j} A_{\lambda_{i}}=\bigcup_{i=1}^{j} D_{i}^{j-1}$ for $j=2, \ldots, n$.

The bouquets $\left(D_{1}^{n}, a\right), \ldots,\left(D_{n+1}^{n}, a\right)$ we define in the following manner:

If $X_{\lambda_{n+1}} \subset A_{\lambda_{n+1}}$, then we put $D_{i}^{n}=D_{i}^{n-1}$ for $i=1, \ldots, n$ and $D_{n+1}^{n}=A_{\lambda_{n+1}}$.

If $X_{\lambda_{n+1}} \not A_{\lambda_{n+1}}$, then $X_{\lambda_{n+1}} \subset \bigcup_{i=1}^{n} D_{l}^{n-1}$ and there is such an $i_{0}$ that $X_{\lambda_{n+1}}$ $\subset D_{i_{0}}^{i-1}$. There is, by (2.3), a subbouquet $\left(F_{n+1}, a\right)$ of the bouquet $\left(D_{l_{0}}^{n-1} \cap Y_{\lambda_{n+1}}, a\right)$ such that $X_{\lambda_{n+1}} \subset F_{n+1}, X_{\lambda_{t_{0}}} \not F_{n+1}$ and the set $\left(D_{i_{0}}^{n-1} \cap Y_{\lambda_{n+1}}-F_{n+1}\right) \cup(a)$ is compact.

We define $D_{i}^{n}=D_{i}^{n-1}$ for $i \neq i_{0}, n+1$, and $D_{i_{0}}^{n}=\left(D_{i_{0}}^{n-1}-F_{n+1}\right) \cup(a)$ and $D_{n+1}^{n}=A_{\lambda_{n+1}} \cup F_{n+1}$.

The bouquets $\left(D_{i}^{j}, a\right)$, where $2 \leqslant j \leqslant n+1, i=1, \ldots, j$, satisfy the conditions (a) $)_{n+1}-(\mathrm{d})_{n+1}$.

The required bouquets $\left(D_{i}, a\right)$ for $i=1, \ldots, k$ we define by the formulas

$$
B_{1}=D_{1}^{k-1} \cup\left(\underset{j \neq n_{1}, \ldots, n_{k}}{\bigcup} A_{\mu_{j}}-\bigcup_{i=2}^{k} D_{i}^{k-1}\right) \text { and } B_{j}=D_{j}^{k-1} \text { for } j=2, \ldots, k \text {. }
$$

It is casy to verify that the bouquets thus defined satisfy conditions (1)-(3) of the lemma.

(2.5) LEMMA. Let $(X, a)$ be a bouquet and $\mathscr{X}=\left\{X_{\lambda}\right\}_{\lambda \in A}$ the family of its leaves. If $\mathscr{U}=\left\{U_{\lambda}\right\}_{\lambda \in \lambda}$ is a family of open subsets of $X$ such that $X_{\lambda} \subset U_{\lambda}$ for every $\lambda \in \Lambda$ and $\lambda_{1}, \ldots, \lambda_{k}$ is a finite sequence of indexes, then there exist finite sequences $\lambda_{k+1}, \ldots, \lambda_{n}$ of indexes and $\left\{\left(B_{i}, a\right)\right\}_{l=1}^{n}$ of subbouquets of $(X, a)$ such that the following conditions are satisfied:

(1) $X_{\lambda_{l}} \subset B_{l} \subset U_{\lambda_{l}}$ for $i=1, \ldots, n$,

(2) $X=\bigcup_{l=1}^{n} B_{l}$

(3) $B_{i} \cap B_{j}=(a)$ for $i \neq j, i, j=1, \ldots, n$.

Proof. We can assume that $X_{\lambda_{1}} \not \subset U_{\lambda_{1}}$ for $i \neq j, i, j=1, \ldots, k$.

There exist, by (2.3), subbouquets $\left(Y_{\lambda_{1}}, a\right), i=1, \ldots, k$, of the bouquet $(X, a)$ such that for every $1 \leqslant i \leqslant k$ the set $\left(X-Y_{\mu_{i}}\right) \cup(a)$ is compact and $X_{\lambda_{i}} \subset Y_{\lambda_{i}} \subset U_{\lambda_{i}}$.

We define $B_{1}=Y_{\lambda_{1}}$ and $B_{i}=\left(Y_{\lambda_{t}}-\bigcup_{j=1} Y_{\lambda_{j}}\right) \cup(d)$ for $i=2, \ldots, k$.

Let us write $X=\left(X-\bigcup_{i=1}^{k} B_{i}\right) \cup(a), \mathscr{M}=\left\{\lambda \in \Lambda: X_{\lambda} \not \subset \bigcup_{i=1}^{k} B_{i}\right\}, V_{\mu}=U_{\mu} \cap \hat{X}$ for $\mu \in \mathscr{M}$ and $\mathscr{V}=\left\{V_{\mu}\right\}_{\mu \in \mathscr{M}}$.

The bouquet $(\mathcal{X}, a)$ and the families $\mathscr{X}=\left\{X_{\mu}\right\}_{\mu \in \mathcal{M}}$ and $\mathscr{V}$ satisfy the conditions of Lemma (2.4). Then if $X \neq(a)$, there exist finite sequences $\lambda_{k+1}, \ldots, \lambda_{n}$ of elements of $\mathscr{M}$ and $\left\{\left(B_{l}, a\right)\right\}_{i=k+1}^{n}$ of subbouquets of $(\mathcal{X}, a)$ such that $\hat{X}=\bigcup_{i=k+1}^{n} B_{i}, X_{\lambda_{i}} \subset B_{i}$ $\subset V_{\lambda_{i}} \subset U_{\lambda_{i}}$ for $i=k+1, \ldots, n$ and $B_{i} \cap B_{j}=(a)$ for $i \neq j, i, j=k+1, \ldots, n$.

All the bouquets $\left(B_{i}, a\right), i=1, \ldots, n$, are subbouquets of $(X, a)$ and satisfy conditions (1), (2) and (3).

(2.6) Theorem. $A$ pointed continuum $(X, a)$ is a bouquet if and only if there is a compact set $Z$ and a family $\mathscr{Z}=\left\{\left(Z_{\mu}, a_{\mu}\right)\right\}_{\mu \in \mathcal{A l}}$ of pointed continua such that the following conditions are satisfied:

(1) for every $\mu \in \mathscr{M}$ the set $Z_{\mu}$ is a component of $Z$,

(2) for every $\mu \in \mathscr{M}$ the set $Z_{\mu}-\left(a_{\mu}\right)$ is connected,

(3) the set $A=\bigcup_{\mu \in \mathbb{A}}\left(a_{\mu}\right)$ is compact,

(4) there exists a continuous map $f:(Z, A) \rightarrow(X, a)$ such that $\left.f\right|_{Z-A}$ is a homeomorphism of the set $Z-A$ onto $X-(a)$.

Proof. First, let us assume that there exist: a compact set $Z$ and a family $\mathscr{Z}$ satisfying conditions (1)-(4) of the theorem.

We define $\Lambda=\left\{\mu \in \mathscr{M}: Z_{\mu}-\left(a_{\mu}\right) \neq \varnothing\right\}$ and $X_{\mu}=f\left(Z_{\mu}\right)$ for every $\mu \in \Lambda$. It is easy to verify that conditions (1)-(3) of definition (1.1) are satisfied.

Now let us suppose that $(X, a)$ is a bouquet and $\mathscr{X}=\left\{X_{\lambda}\right\}_{\lambda \in A}$ is the family of its leaves.

For $i=1,2, \ldots$ let us denote by $\mathscr{A}_{i}=\left\{A_{1}^{i}, \ldots, A_{k_{i}}^{i}\right\}$ a finite family of closed subsets of $X$ such that 
(i) $X=\bigcup_{j=1}^{k_{l}} A_{j}^{l}$ for $l=1,2, \ldots$,

(ii) $A_{j}^{l} \cap A_{j^{\prime}}^{l}=(a)$ for $j \neq j^{\prime}, j, j^{\prime}=1, \ldots, k_{l}, l=1,2, \ldots$,

(iii) $\left(A_{j}^{l}, a\right)$ is a subbouquet of $(X, a)$ for $j=1, \ldots, k_{l}, l=1,2, \ldots$

It follows from Lemma (2.5) that we can find the sequence $\mathscr{A}_{1}, \mathscr{A}_{2}, \ldots$ in such a manner that the following conditions are satisfied:

$(\alpha)$ for every $A \in \mathscr{A}_{i}$ there is $B \in \mathscr{A}_{i-1}$ such that $A \subset B$,

(B) for every $A \in \mathscr{A}_{i}$ there is an index $\lambda(A) \in \Lambda$ such that $A \subset K\left(X_{\lambda(A)}, 1 / i\right)$.

We denote by $B_{1}^{n}, \ldots, B_{k_{n}}^{n}$ a sequence of closed subsets of the Hilbert cube $Q$ such that the set $B_{j}^{n}$ is homeomorphic to $A_{j}^{n}$ for $j=1, \ldots, k_{n}$ and $B_{j}^{n} \cap B_{i}^{n}=\varnothing$ for $i \neq j, i, j=1, \ldots, k_{n}$.

For every natural $n$ the set $Y_{n}=\bigcup_{j=1}^{k_{n}} B_{i}^{n}$ is compact and there is a continuous map $g_{n}: Y_{n} \rightarrow X$ such that $\left.g_{n}\right|_{B_{j}^{n}}$ is a homeomorphism of $B_{j}^{n}$ onto $A_{j}^{n}$ for $j=1, \ldots, k_{n}$.

It follows from conditions $(\alpha)$ and (i)-(iii) that for every $j \in\left\{1, \ldots, k_{n+1}\right\}$ there exists exactly one number $l_{j}^{n} \in\left\{1, \ldots, k_{n}\right\}$ such that $A_{j}^{n+1} \subset A_{l_{j}}^{n}$.

The maps $p_{n}^{n+1}: Y_{n+1} \rightarrow Y_{n}$ and $p_{n}^{n^{\prime}}: Y_{n^{\prime}} \rightarrow Y_{n}$ defined by the formulas

$$
p_{n}^{n+1}(x)=\left(\left.g_{n}\right|_{B_{j}^{n}}\right)^{-1} g_{n+1}(x) \quad \text { for } \quad x \in B_{j}^{n+1}, j=1, \ldots, k_{n+1}
$$

and

$$
p_{n}^{n^{\prime}}=p_{n}^{n+1} \ldots p_{n^{\prime}-1}^{n^{\prime}} \quad \text { for } \quad n^{\prime}>n
$$

are continuous.

Let us write $Z=\varliminf_{\{}\left\{Y_{n}, p_{n^{n}}^{n}, N\right\}$ and denote by $p_{n}: Z \rightarrow Y_{n}$ the natural projection and by $p_{0}: Z \rightarrow X$ the map defined by the formula

$$
p_{0}(z)=g_{1} p_{1}(z) \quad \text { for every } z \in Z \text {. }
$$

Let us observe that if $\lambda, \mu \in \Lambda$ and $\lambda \neq \mu$, then the sets $p_{0}^{-1}\left(X_{\lambda}\right)$ and $p_{0}^{-1}\left(X_{\mu}\right)$ lie in different components of the space $Z$ and for every component $S$ of $Z$ the intersection $S \cap p_{0}^{-1}(a)$ contains only one point.

Let $\mathscr{Z}=\left\{\left(Z_{\mu}, a_{\mu}\right)\right\}_{\mu_{\in} M}$ be the family of pointed components of $Z$, where $a_{\mu}$ is the point in $Z_{\mu} \cap p_{0}^{-1}(a)$ for $\mu \in \mathscr{M}$, and we can assume that $Z_{\mu^{\prime}} \neq Z_{\mu}$ if $\mu^{\prime} \neq \mu$, $\mu^{\prime}, \mu \in \mathscr{M}$.

The map $f:(Z, A) \rightarrow(X, a)$ defined by the formula

$$
f(z)=p_{0}(z) \quad \text { for } \quad z \in Z,
$$

where $A=\bigcup_{\mu \in \mathscr{M}}\left(a_{\mu}\right)$, is continuous and satisfies the required conditions. Indeed, the map $g: X \in \mathscr{M}$ : $X(a) \rightarrow Z-A$, where $g(x)=\left(g_{1}^{-1}(x),\left(g_{1} p_{1}^{2}\right)^{-1}(x), \ldots\right)$ for every $x \in X-(a)$, is continuous and it is the inverse of $\left.f\right|_{Z-A}$.

\section{Some properties of bouquets.}

(3.1) If a pointed continuum $(X, a)$ is a bouquet and $\mathscr{X}=\left\{X_{\lambda}\right\}_{\lambda \in \Lambda}$ is the family of its leaves, then $\operatorname{dim} X=\operatorname{supdim} X_{\lambda}$.
Proof. It is clear that if $\operatorname{supdim} X_{\lambda}=\infty$, then $\operatorname{dim} X=\infty$. $\lambda \in A$

Let us suppose that there is a natural number $n$ such that $\sup \operatorname{dim} X_{\lambda}=n$. Let us write $Y_{m}=X-K(a, 1 / m)$ for $m=1,2, \ldots$ If $Y_{m}=\emptyset$, then $\operatorname{dim} X_{m}$ $=-1<n$, and if $Y_{m} \neq \varnothing$, then since the dimension of each component of $Y_{m}$ is not greater than $n$, we infer ([8], p. 90) that $\operatorname{dim} Y_{m} \leqslant n$.

It is clear that $X=(a) \cup \bigcup_{k=1}^{\infty} Y_{k}$, and so we infer ([8], p. 30) that $\operatorname{dim} X \leqslant n$.

Since $X$ contains an $n$-dimensional subset, we have $\operatorname{dim} X=n$.

It is easy to observe that

(3.2) If $(X, a)$ is a bouquet, $\mathscr{X}=\left\{X_{\lambda}\right\}_{\lambda \in \Lambda}$ is the family of its leaves and $h: X \rightarrow Y$ is a homeomorphism, then $(Y, h(a))$ is a bouquet and $\mathscr{Y}=\left\{h\left(X_{\lambda}\right)\right\}_{\lambda \in A}$ is the family of its leaves.

It follows directly from (3.2) that if $(X, a)$ and $(Y, b)$ are homeomorphic bouquets, $\mathscr{X}=\left\{X_{\lambda}\right\}_{\lambda \in \Lambda}$ and $\mathscr{Y}=\left\{Y_{\mu}\right\}_{\mu \in \mathcal{M}}$ are the families of leaves of $(X, a)$ and $(Y, b)$, respectively, then there is a one-to-one map $\varphi: \mathscr{X} \rightarrow \mathscr{Y}$ such that for every $\lambda \in \Lambda$ the pointed continuum $\left(X_{\lambda}, a\right)$ is homeomorphic to $\left(\varphi\left(X_{\lambda}\right), b\right)$.

(3.3) Diminition. A bouquet $(X, a)$ is said to be disperse if for every leaf $X_{\lambda}$ of $(X, a)$ the set $X_{\lambda}-(a)$ is open in $X$.

Let us observe that a disperse bouquet has a countable family of leaves.

(3.4) Throrem. Let $(X, a)$ be a bouquet and $\mathscr{X}=\left\{X_{\lambda}\right\}_{\lambda \in A}$ the family of its leaves. Then $(X, a)$ is disperse if and only if for every $\varepsilon>0$ the condition $\delta\left(X_{\lambda}\right)<\varepsilon$ is satisfied for almost all $\lambda$.

Proof. Let us suppose that the bouquet $(X, a)$ is disperse and $\varepsilon$ is a positive number. It is clear that there exists a finite sequence $\lambda_{1}, \ldots, \lambda_{k}$ of indexes such that $X=K\left(a, \frac{1}{2} \varepsilon\right) \cup \bigcup_{l=1}^{k} X_{\lambda_{l}}-(a)$. Then $K\left(a, \frac{1}{2} \varepsilon\right)$ contains almost all leaves $X_{\lambda}$.

Now let us suppose that for every $\varepsilon>0$ the condition $\delta\left(X_{\lambda}\right)<\varepsilon$ is satisfied for almost all $X_{\lambda} \in \mathscr{X}$.

Let $X_{\lambda} \in \mathscr{X}$ be any fixed leaf, $x \in X_{\lambda}-(a)$ a fixed point and $\varepsilon>0$ a number such that $\varrho(x, a)>2 \varepsilon$. Let $\lambda_{1}, \ldots, \lambda_{k}$ be all indexes in $A$ such that $X_{\lambda_{j}} \neq K(a, \varepsilon)$ and $\lambda_{j} \neq \lambda$ for $j=1, \ldots, k$. The number $r=\min \left(\varepsilon, \varrho\left(x, X_{\lambda_{l}}\right), \ldots, \varrho\left(x, X_{\lambda_{k}}\right)\right)$ is greater than 0 and $K(x, r) \cap X_{\mu}=\Theta$ for $\mu \neq \lambda$. Then $X_{\lambda}-(a)$ is a neighborhood of $x$ in $X$.

Then the bouquet $(X, a)$ is disperse.

(3.5) THorrim. If $(X, a)$ and $(Y, b)$ are disperse bouquets and $\mathscr{X}=\left\{X_{\lambda}\right\}_{\lambda \in A}$, $\mathscr{Y}=\left\{Y_{\mu}\right\}_{\mu \in \mathbb{N l}}$ are the fumilies of leaves of $(X, a)$ and $(Y \quad b)$, respectively, then $(X, a)$ is homeomorphic to $(Y, b)$ if and only if there exists a one-to-one map $\varphi: A \rightarrow \mathscr{M}$ such that for every $\lambda \in \Lambda$ the pointed continua $\left(X_{\lambda}, a\right)$ and $\left(Y_{\varphi(\lambda)}, b\right)$ are homeomorphic.

Proof. Let us assume that there exists a one-to-one map $\varphi: \Lambda \rightarrow M$ such that for every $\lambda \in \Lambda$ there is a homeomorphism $h_{\lambda}:\left(X_{\lambda}, a\right) \rightarrow\left(Y_{\varphi(\lambda)}, b\right)$. 
It is easy to verify that the map $h:(X, a) \rightarrow(Y, b)$ defined by the formula

$$
h(x)=h_{\lambda}(x) \text { for every } x \in X_{\lambda} \text { and } \lambda \in \Lambda
$$

is a homeomorphism.

The second part of the theorem we obtain from (3.2)

(3.6) If $(X, a)$ is a disperse bouquet, then each leaf of $(X, a)$ is a retract of the space $X$.

Proof. Let $X_{\lambda}$ be any leaf of $(X, a)$. Since the set $X-\left(X_{\lambda}-(a)\right)$ is closed in $X$, the formula

$$
r_{\lambda}(x)=\left\{\begin{array}{lll}
x & \text { if } & x \in X_{\lambda} \\
a & \text { if } & x \in\left(X-X_{\lambda}\right) \cup(a)
\end{array}\right.
$$

defines the retraction $r_{\lambda}: X \rightarrow X_{\lambda}$.

(3.7) If $(X, a)$ is a disperse bouquet, then $X$ is locally connected if and only if each leaf of $(X, a)$ is locally connected.

Proof. Let $\mathscr{X}=\left\{X_{\lambda}\right\}_{\lambda \in A}$ be the family of leaves of $(X, a)$.

If $X$ is locally connected, then it follows from (3.6) that for every $\lambda \in \Lambda$ the leaf $X_{\lambda}$ has the same property.

Now let us assume that each leaf of $(X, a)$ is locally connected.

If $x \in X-(a)$ is any point, then $x \in X_{\lambda}-(a)$ for some $\lambda \in \Lambda$. Since the set $X_{\lambda}-(a)$ is open in $X_{2}$ and in $X$ and $X_{\lambda}$ is locally connected, we infer that $X$ is locally connected at the point $x$.

Let $\varepsilon$ be any positive number and let $\lambda_{1}, \ldots, \lambda_{k}$ be all the indexes such that $X_{\lambda_{i}} \notin K(a, \varepsilon)$. There are connected sets $U_{1}, \ldots, U_{k}$ such that for every $1 \leqslant i \leqslant k$ the set $U_{i}$ is a neighborhood of $a$ in $X_{\lambda_{t}}$ and $U_{i} \subset K(a, \varepsilon)$.

The set $U=\bigcup_{i=1}^{k} U_{i} \cup \bigcup_{\lambda \neq \lambda_{1}, \ldots, \lambda_{k}} X_{\lambda}$ is a connected neighborhood of $a$ in $X$ contained in $K(a, \varepsilon)$. Thus $X$ is also locally connected at $a$.

(3.8) If $(Y, a)=\left\{\left(Y_{n}, a\right), p_{n}^{n^{\prime}}, N\right\}$ is an inverse sequence of finite bouquets lying in the Hilbert cube and if, for every natural $n, Y_{n} \subset Y_{n+1}$ and $p_{n}^{n+1}: Y_{n+1} \rightarrow Y_{n}$ is a retraction such that $p_{n}^{n+1}\left(Y_{n+1}-Y_{n}\right)=(a)$, then the inverse limit $(Y, \hat{a})$ of $(Y, a)$ is a disperse bouquet and there exists a one-to-one map $\varphi$ of the family of of leaves of $(Y, \hat{a})$ onto the sum $\bigcup_{i=1}^{\infty} \mathscr{Y}_{n}$ of the families of leaves of $\left(Y_{n}, a\right)$, for $n=1,2, \ldots$, such that every leaf $Z \in \mathscr{O}$ is homeomorphic to $\varphi(Z)$.

Proof. We can assume that $\mathscr{Y}_{n}=\left\{X_{1}, \ldots, X_{n}\right\}$.

Let $i_{n}^{n^{\prime}}:\left(Y_{n}, a\right) \rightarrow\left(Y_{n^{\prime}}, a\right)$, for $n^{\prime}>n$, be the inclusion, and $i_{n}:\left(Y_{n}, a\right) \rightarrow(Y, \hat{a})$ the map defined by the formula:

$$
\begin{aligned}
& i_{n}(y)=\left\{\begin{array}{cc}
(\underbrace{a, \ldots, a}_{n-1}, y, y, \ldots) & \text { for } y \in Y_{n}-Y_{n-1} \\
i_{n-1}(y) & \text { for } y \in Y_{n-1}
\end{array} \text { for } n>1\right. \\
& i_{1}(y)=(y, y, \ldots) \text { for } y \in Y_{1}
\end{aligned}
$$

and let $p_{n}:(Y, \hat{a}) \rightarrow\left(Y_{n}, a\right)$ be the projection of the inverse limit $(n \geqslant 1)$.
It is clear that for every natural $n$ the set $Z_{n}=i_{n}\left(X_{n}\right)$ is homeomorphic to $X_{n}$. Let $y=\left(y_{1}, y_{2}, \ldots\right) \in X$ be an arbitrary point.

If $y \neq \hat{a}=(a, a, \ldots)$, then there is an $n_{0}$ such that $y_{n}=y_{n_{0}} \neq a$ for $n \geqslant n_{0}$ and $y_{n}=a$ for $n<n_{0}$. Thus $y_{n_{0}} \in X_{n_{0}}$ and $y=i_{n_{0}}\left(y_{n_{0}}\right) \in \bigcup_{n=1}^{\infty} Z_{n}$. If $y=\hat{a}$, then evidently $y \in \bigcup_{n=1}^{\infty} Z_{n}$.

Then $X=\bigcup_{n=1}^{\infty} Z_{n}$.

Since the set $Z_{n}-(\hat{a})$ is homeomorphic to $X_{n}-(a)$, it is nonempty and connected.

Let $n$ and $m$ be fixed natural numbers and $n>m$. The sets $C_{m}=\bigcup_{j=1}^{n-1} Z_{j}$ and $C_{n}=\bigcup_{j=n}^{\infty} Z_{j}$ are continua $\left(C_{n}\right.$ is homeomorphic to the inverse limit of the inverse

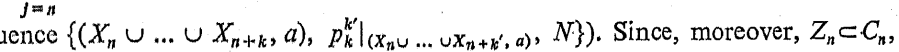
$Z_{m} \subset C_{m}, Y=C_{n} \cup C_{m}$ and $C_{n} \cap C_{m}=(\hat{a})$, we infer that $(Y, \hat{a})$ is a bouquet and the sets $Z_{n}$, for $n \in N$, are leaves of $(Y, \hat{a})$.

The bouquet $(Y, \hat{a})$ is disperse, because for every $Z_{n}$ the set $Z_{n}-(\hat{a})=Y-$ $-\left(\bigcup_{j=1}^{n-1} Z_{j} \cup \bigcup_{j=n+1}^{\infty} Z_{j}\right)$ is an open subset of $Y$. The function $\varphi: \mathscr{Y} \rightarrow \bigcup_{n=1}^{\infty} \mathscr{Y}_{n}$ defined by $\varphi\left(Z_{n}\right)=X_{n}$ satisfies the required conditions.

(3.9) A leaf of a bouquet $(X, a)$ is a retract of the space $X$ if and only if it is a neighborhood retract of $X$.

Proof. If the leaf $X_{\lambda}$ is a retract of $X$, then it is evidently a neighborhood retract of $X$.

Now let us assume that there are: a neighborhood $U$ of $X_{\lambda}$ in $X$ and a retraction $\hat{r}: U \rightarrow X_{\lambda}$. Let $(B, a)$ be a subbouquet of $(X, a)$ satisfying the conditions of Lemma (2.3). The formula

$$
r(x)=\left\{\begin{array}{cll}
\hat{r}(x) & \text { if } & x \in B \\
a & \text { if } & x \in(X-B) \cup(a)
\end{array}\right.
$$

defines a retraction $r: X \rightarrow X_{\lambda}$.

It follows from (3.9) that

(3.10) If $X_{2} \in$ ANR is a leaf of a bouquet $(X, a)$, then it is a retract of the space $X$.

(3.11) ExAMPLis. Let us write

$P_{0}=\left\{(x, y, z) \in E^{3}: z=0\right\}$,

$P_{n}=\left\{(x, y, z) \in E^{3}: x+y-n z=0\right\}$ for $n=1,2, \ldots$,

$I=\left\{(x, y, z) \in E^{3}: x=0\right.$ and $\left.0 \leqslant y \leqslant 2\right\}$,

$A_{0}=\left\{(x, y, z) \in E^{3}: 0<x \leqslant 1\right.$ and $\left.y=\sin \frac{1}{x}\right\}$, 
$A_{n}=\left\{(x, y, z) \in E^{3}: \frac{1}{n \pi} \leqslant x \leqslant 1\right.$ and $\left.y=1+\sin \frac{1}{x}\right\}$ for $n=1,2, \ldots$,

$B_{n}=\left\{(x, y, z) \in E^{3}: y=1\right.$ and $\left.0 \leqslant x \leqslant \frac{1}{n \pi}\right\}$ for $n=1,2, \ldots$,

$Y_{n}=I \cup A_{n} \cup B_{n}$ for $n=1,2, \ldots, Y_{0}=I \cup A_{0}, X_{n}=P_{n} \cap Y_{n}$ for $n=1,2, \ldots$, $X=\bigcup_{n=1}^{\infty} X_{n}$.

It is easy to verify that the pointed continum $(X,(0,0,0))$ is a bouquet, $X$ $=\left\{X_{n}\right\}_{n=0}^{\infty}$ is the family of its leaves and the leaf $X_{0}$ is not a retract of the space $X$.

4. On movability of bouquets. K. Borsuk introduced ([4], p. 223) an important notion of movability of pointed compacta.

(4.1) Definition ([4], p. 223). A pointed compactum $\left(X, x_{0}\right)$ lying in the pointed Hilbert cube $\left(Q, x_{0}\right)$ is said to be movable if for every neighborhood $U$ of $X$ there is a neighborhood $V$ of $X$ such that for every neighborhood $W$ of $X$ there is a homotopy $\varphi: V \times\langle 0,1\rangle \rightarrow U$ such that $\varphi(x, 0)=1$ and $\varphi(x, 1) \in W$ for every point $x \in V$ and $\varphi\left(x_{0}, t\right)=x_{0}$ for every $0 \leqslant t \leqslant 1$.

The following question arises: what is the relation between the movability of a bouquet and the movability of its leaves? In order to answer it, let us observe that:

(4.2) If $X$ is a closed subset of the Hilbert cube $Q, x_{0} \in X$ is a fixed point and for every $\varepsilon>0$ there is a closed subset $A_{0}$ of $X$ containing $x_{0}$ such that $\left(A_{B}, x_{0}\right)$ is movable and there is a continuous map $f_{\varepsilon}:\left(X, x_{0}\right) \rightarrow\left(A_{\varepsilon}, x_{0}\right)$ satisfying the condition $\varrho\left(f_{\varepsilon}(x), x\right)<\varepsilon$ for every $x \in X$, then $\left(X, x_{0}\right)$ is movable.

(4.3) LEMMA. Let $(X, a)$ be a bouquet and $\mathscr{X}=\left\{X_{\lambda}\right\}_{\lambda \in A}$ the family of its leaves. If for every $\lambda \in \Lambda$ the leaf $X_{\lambda}$ is a retract of $X$, then for every $\varepsilon>0$ there is a finite sequence $X_{\lambda_{1}}, \ldots, X_{\lambda_{n}}$ of leaves and there is a retraction $r_{\varepsilon}: X \rightarrow \bigcup_{i=1}^{n} X_{\lambda_{i}}$ satisfying the condition $\varrho\left(r_{\varepsilon}(x), x\right)<\varepsilon$ for every $x \in X$.

Proof. For every $\lambda \in \Lambda$ there is a neighborhood $U_{\lambda}$ of $X_{\lambda}$ in $X$ and a retraction $q_{\lambda}: U_{\lambda} \rightarrow X_{\lambda}$ such that $\varrho\left(q_{\lambda}(x), x\right)<\varepsilon$ for every $x \in U_{\lambda}$.

There are finite sequences $X_{\lambda_{1}}, \ldots, X_{\lambda_{n}}$ of leaves and $\left\{\left(B_{i}, a\right)\right\}_{i=1}^{n}$ of subbouquets of $(X, a)$ satisfying conditions (1)-(3) of Lemma (2.4).

The map $r_{\varepsilon}: X \rightarrow \bigcup_{i=1}^{n} X_{\lambda_{i}}$ defined by the formula

$$
r_{\varepsilon}(x)=q_{\lambda_{i}}(x) \quad \text { for } \quad x \in B_{i}, i=1, \ldots, n
$$

is the required retraction.

From Lemmas (4.2), (4.3) and Borsuk's theorem ([4], p. 224, Th. (2.8)) results the following

(4.4) TheOREM. Let $(X, a)$ be a bouquet and $\mathscr{X}=\left\{X_{\lambda}\right\}_{\lambda \in A}$ the family of its leaves.
If for every $\lambda \in \Lambda$ the leaf $X_{\lambda}$ is a retract of $X$ and $\left(X_{\lambda}, a\right)$ is movable, then $(X, a)$ is also movable.

Particularly we get

(4.5) Corollary. If $(X, a)$ is a bouquet and each of its leaves is an ANR-set, then $(X, a)$ is movable.

The movability of a bouquet does not imply the movability of its leaves.

(4.6) ExAMPLE. Let $S_{1}, S_{2}, \ldots$ be a sequence of 1-spheres such that $S_{i} \cap S_{j}$ $=(a)$ for $i \neq j, i, j=1,2, \ldots$ and let $\alpha_{n}^{n+1}:\left(S_{n+1}, a\right) \rightarrow\left(S_{n}, a\right), n=1,2, \ldots$ be continuous maps with the degree 2. Let $X_{n}=S_{1} \cup \ldots \cup S_{n}, n=1,2, \ldots$, and $p_{n}^{n+1}:\left(X_{n+1}, a\right) \rightarrow\left(X_{n}, a\right)$ be the continuous map defined by the formula

$$
p_{n}^{n+1}(x)=\left\{\begin{array}{ccl}
\alpha_{n}^{n+1}(x) & \text { if } & x \in S_{n+1}, \\
x & \text { if } & x \in S_{1} \cup \ldots \cup S_{n} .
\end{array}\right.
$$

The pointed continuum $(X, \hat{a})=\underline{\operatorname{Lim}}\left\{\left(X_{n}, a\right), p_{n}^{n^{\prime}}, N\right\}$ is movable ([11], pp. 250-252).

It is easy to verify that $(X, a)$ is a bouquet and the set $S=\operatorname{Lim}\left\{S_{n}, \alpha_{n}^{n^{\prime}}, N\right\}$ is one of the leaves of $(X, \hat{a})$.

It is known ([3], p. 138) that $S$ is not movable.

(4.7) Theorem. Let $(X, a)$ be a bouquet and $X=\left\{X_{\lambda}\right\}_{\lambda \in \Lambda}$ the family of its leaves. Then $\operatorname{Sh}(X, a)=\operatorname{Sh}(a, a)$ if and only if $\operatorname{Sh}\left(X_{\lambda}, a\right)=\operatorname{Sh}(a, a)$ for every $\lambda \in \Lambda$.

Proof. It is known ([6], Chapter VIII, Corollaries (4.6) and (4.7)) and comp. ([2], pp. 72-73, Th. (9.1) and (9.8)) that the following conditions are equivalent:

(1) $X$ is an FAR - set,

(2) for every $a \in X$ and for every neighborhood $U$ of $X$ in $Q$ there is a continuous map $\varphi: X \times\langle 0,1\rangle \rightarrow U$ such that $\varphi(x, 0)=x$ and $\varphi(x, 1)=a$ for every $x \in X$,

(3) for every $a \in X$ and every neighborhood $U$ of $X$ in $Q$ there are: a neighborhood $V$ of $X$ in $Q$ and a continuous map $\varphi: V \times\langle 0,1\rangle \rightarrow U$ such that $\varphi(x, 0)$ $=x, \varphi(x, 1)=a$ for every $x \in V$ and $\varphi(a, t)=a$ for every $t \in\langle 0,1\rangle$,

(4) $\operatorname{Sh}(X, a)=\operatorname{Sh}(a, a)$.

Let us assume that $\operatorname{Sh}(X, a)=\operatorname{Sh}(a, a)$. Let $X_{\lambda}$ be a fixed leaf and let $W$ be an open neighborhood of $X_{\lambda}$ in $Q$. The set $U=W \cap X$ is a neighborhood of $X_{2}$ in $X$. There is subbouquet $(Y, a)$ of $(X, a)$ satisfying the conditions of Lemma (2.3).

The map $r: X \rightarrow Y$ defined by the formula

$$
r(x)=\left\{\begin{array}{lll}
x & \text { if } & x \in Y \\
a & \text { if } & x \in(X-Y) \cup(a)
\end{array}\right.
$$

is a retraction.

Then the set $Y$ is an FAR-set and there is a continuous map $\psi: Y \times\langle 0,1\rangle \rightarrow U$ such that $\psi(x, 0)=x, \psi(x, 1)=a$ for every $x \in Y$ and $\psi(a, t)=a$ for every $t \in\langle 0,1\rangle$.

The map $\varphi: X_{2} \times\langle 0,1\rangle \rightarrow U$ defined by the formula

$$
\varphi(x, t)=\psi(x, t) \text { for every } x \in X_{\lambda} \text { and } t \in\langle 0,1\rangle \text {. }
$$

is continuous and condition (2) is satisfied / $/ 4$

2-- Fundamenta Mathematleae XCrI 
Then $\operatorname{Sh}\left(X_{\lambda}, a\right)=\operatorname{Sh}(a, a)$.

Now let us assume that $\operatorname{Sh}\left(X_{\lambda}, a\right)=\operatorname{Sh}(a, a)$ for every $\lambda \in A$ and let $U$ be an arbitrary neighborhood of $X$ in $Q$.

For every $\lambda \in \Lambda$ there are: a neighborhood $U_{\lambda}$ of $X_{\lambda}$ in $Q$ and a continuous map $\varphi_{\lambda}: U_{\lambda} \times\langle 0,1\rangle \rightarrow U$ such that $\varphi_{\lambda}(x, 0)=x, \varphi_{\lambda}(x, 1)=a$ for every $x \in U_{\lambda}$ and $\varphi_{\lambda}(a, t)=a$ for every $t \in\langle 0,1\rangle$.

There are finite sequences: $\lambda_{1}, \ldots, \lambda_{n}$ of indexes and $\left\{\left(B_{i}, a\right)\right\}_{i=1}^{n}$ of subbouquets of $(X, a)$ satisfying conditions (1)-(3) of (2.4).

The map $\varphi: X \times\langle 0,1\rangle \rightarrow U$ defined by the formula

$$
\varphi(x, t)=\varphi_{\lambda_{i}}(x, t) \quad \text { if } \quad x \in B_{i}, t \in\langle 0,1\rangle, \quad i=1, \ldots, n
$$

is continuous and satisfies the conditions: $\varphi(x, 0)=x, \varphi(x, 1)=a$ for every $x \in X$ and $\varphi(a, t)=a$ for every $t \in\langle 0,1\rangle$.

Then condition (2) is satisfied and $\operatorname{Sh}(X, a)=\operatorname{Sh}(a, a)$.

5. Bouquets and inverse sequences of finite bouquets. It is convenient in the theory of shape to consider continua as inverse limits of inverse ANR - sequences ([9], pp. 41-42).

We shall prove that some bouquets are homeomorphic to inverse limits of inverse sequences of finite bouquets.

(5.1) LEMMA. Let $(X, a)$ be a bouquet and $\mathscr{X}=\left\{X_{\lambda}\right\}_{\lambda \in A}$ the family of its leaves. If for every $\lambda \in \Lambda$ the leaf $X_{\lambda}$ is a retract of $X$, then there are sequences $\lambda_{1}, \lambda_{2}, \ldots$ of indexes and $k_{1} \leqslant k_{2} \leqslant \ldots$ of natural numbers such that for every $n$ there exists a retraction $r_{n}: X \rightarrow \bigcup_{i=1}^{k_{n}} X_{\lambda_{i}}$ satisfying the condition $\varrho\left(x, r_{n}(x)\right)<1 / n$ for every $x \in X$.

Proof. If $q_{\lambda}: X \rightarrow X_{\lambda}$, for $\lambda \in \Lambda$, denotes a retraction, then there are neighborhoods $W_{\lambda}^{1}$, for $\lambda \in \Lambda$, such that $\varrho\left(q_{\lambda}(x), x\right)<1$ for every $x \in W_{\lambda}^{1}$.

There exist finite sequences $\lambda_{1}, \ldots, \lambda_{k_{1}}$ of indexes and $\left\{\left(B_{i}^{1}, a\right)\right\}_{i=1}^{k_{1}}$ of subbouquets of $(X, a)$ satisfying conditions (1)-(3) of (2.4).

The formula

$$
r_{1}(x)=q_{\lambda_{i}}(x) \text { for every } x \in B_{i}^{1} \text { and } i=1, \ldots, k_{1}
$$

defines a retraction $r_{1}: X \rightarrow \bigcup_{i=1}^{k_{1}} X_{\lambda_{i}}$.

Let as assume that we have defined natural numbers $k_{1} \leqslant \ldots \leqslant k_{n-1}$, leaves $X_{\lambda_{1}}, \ldots, X_{\lambda_{k_{n-1}}}$ and retractions $r_{i}: X \rightarrow \bigcup_{j=1}^{k_{i}} X_{\lambda_{j}}, i=1, \ldots, n-1$.

Let $W_{\lambda}^{n}$, for $\lambda \in \Lambda$, be a neighborhood of $X_{\lambda}$ in $X$ such that $\varrho\left(q_{\lambda}(x), x\right)<1 / n$ for every point $x \in W_{\lambda}^{n}$.

If $X-\bigcup_{j=1}^{k_{n}-1} X_{\lambda_{j}} \neq \varnothing$, then there are: a natural number $k_{n} \geqslant k_{n-1}$ and sequences $\lambda_{k_{n-1}+1}, \ldots, \lambda_{k_{n}}$ of indexes and $\left\{\left(B_{i}^{n}, a\right)\right\}_{i=1}^{k_{n}}$ of subbouquets of $(X, a)$ satisfying. conditions (1)-(3) of (2.5).
The formula

$$
r_{n}(x)=q_{\lambda_{i}}(x) \text { for } \quad x \in B_{i}^{n}, i=1, \ldots, k_{n},
$$

defines the required retraction $r_{n}: X \rightarrow \bigcup_{i=1}^{k_{n}} X_{\lambda_{i}}$.

(5.2) LEMMA. If $X$ is a continuum, $A_{1} \subset A_{2} \subset \ldots$ is a sequence of subsets of $X$ such that for every $n$ there is a retraction $r_{n}: X \rightarrow A_{n}$ satisfying the condition $\varrho\left(r_{n}(x), x\right)<1 / n$ for every $x \in X$, then there is a sequence $k_{1}<k_{2}<\ldots$ of natural numbers such that the space $X$ is homeomorphic to $\underline{\operatorname{Lim}}\left\{Y_{n}, p_{n}^{n^{\prime}}, N\right\}$, where $Y_{n}=A_{k_{n}}$ and $p_{n}^{n+1}=\left.r_{k_{n}}\right|_{A_{k_{n+1}}}$.

Proof. We define $k_{1}=1$.

Let $k_{2} \geqslant 4$ be a natural number such that

$$
\varrho\left(r_{1} r_{k_{2}}(x), r_{1}(x)\right)<2^{-2} \quad \text { for every } x \in X .
$$

Let us assume that we have defined a sequence $k_{1}<k_{2}<\ldots<k_{n-1}$ of natural numbers such that $k_{i} \geqslant i^{2}$ and

$\varrho\left(r_{k_{i}} \ldots r_{k_{j-1}} r_{k_{J}}(x), r_{k_{i}} \ldots r_{k_{j-1}}(x)\right)<j^{-2}$ for every $x \in X$ and $i=1,2, \ldots, n-1$.

There is a natural number $k_{n}>\max \left(k_{n-1}, n^{2}\right)$ such that

$\varrho\left(r_{k_{i}} \ldots r_{k_{n-1}} r_{k_{n}}(x), r_{k_{j}} \ldots r_{k_{n-1}}(x)\right)<n^{-2} \quad$ for every $x \in X$ and $j=1, \ldots, n-1$.

We shall prove that $X$ is homeomorphic to $Y=\operatorname{Lim}\left\{Y_{n}, p_{n}^{n^{\prime}}, N\right\}$, where $Y_{n}=A_{k_{n}}, p_{n}^{n+1}=\left.q_{n}\right|_{Y_{n+1}}$ and $q_{n}=r_{k_{n}}$ for $n=1,2, \ldots$

We define the metric $\hat{\varrho}$ in ${\stackrel{\boldsymbol{P}}{\boldsymbol{P}} Y_{n}}_{n}$ by the formula

$$
\hat{\varrho}(x, y)=\sum_{n=1}^{\infty} 2^{-n} \varrho\left(x_{n}, y_{n}\right) \quad \text { for every } x=\left(x_{n}\right)_{n=1}^{\infty}, y=\left(y_{n}\right)_{n=1}^{\infty} \in \underset{n=1}{\boldsymbol{P}} Y_{n} .
$$

The maps $h_{n}: X \rightarrow \underset{k=1}{\boldsymbol{P}} Y_{k}$, for $n=1,2, \ldots$, defined by the formulas

$$
h_{n}(x)=\left(q_{1} \ldots q_{n-1} q_{n}(x), q_{2} \ldots q_{n-1} q_{n}(x), \ldots, q_{n-1} q_{n}(x), q_{n}(x), q_{n+1}(x), \ldots\right)
$$

are continuous.

Since

$$
\hat{\varrho}\left(h_{n}(x), h_{n+1}(x)\right)<n^{-2} \quad \text { for every } x \in X \text { and } n=1,2, \ldots,
$$

there exists a continuous map $h: X \rightarrow \underset{k=1}{\infty} Y_{k}$ such that $h=\lim _{n \rightarrow \infty} h_{h}$.

We shall prove that $h$ leads $X$ homeomorphically onto $Y$. .

If $x \in X$, then $h(x)=\left(x_{1}, x_{2}, \ldots\right)$, where $x_{k}=\lim _{n \rightarrow \infty} q_{k} \ldots q_{n}(x)$; hence

$$
p_{k}^{k+1}\left(x_{k+1}\right)=p_{k}^{k+1}\left(\lim _{n \rightarrow \infty} q_{+1} \ldots q_{n}(x)\right)=\lim _{n \rightarrow \infty} p_{k}^{k+1}\left(q_{k+1} \ldots q_{n}(x)\right)=x_{k},
$$

and thus $h(x) \in Y$.

2* 
If $y \in Y$, then $y=\left(y_{1}, y_{2}, \ldots\right)$ and $y_{n}=p_{n}^{n+1}\left(y_{n+1}\right)$ for $n=1,2, \ldots$ There is a $y_{0} \in X$ such that $y_{0}=\lim _{n \rightarrow \infty} y_{n}$, because $\varrho\left(y_{n}, y_{n+1}\right)<n^{-2}$ for every $n$ and $X$ is compact.

The formuila

$$
g\left(y_{1}, y_{2}, \ldots\right)=\lim _{n \rightarrow \infty} y_{n} \quad \text { for every }\left(y_{1}, y_{2}, \ldots\right) \in Y
$$

defines the map $g: Y \rightarrow X$.

It is easy to verify that

$$
\begin{array}{ll}
g h(x)=x & \text { for every } x \in X, \\
h g(y)=y & \text { for every } y \in X .
\end{array}
$$

Then the continuous map $h: X \rightarrow Y$ is a homeomorphism of $X$ onto $Y$.

Lemmas (5.1) and (5.2) directly imply

(5.3) THEOREM. Let $(X, a)$ be a bouquet and $\mathscr{X}=\left\{X_{\lambda}\right\}_{\lambda \in A}$ the family of its leaves. If for every $\lambda \in \Lambda$ the leaf $X_{\lambda}$ is a retract of $X$, then the bouquet $(X, a)$ is homeomorphic to $(Y, \hat{a})=\operatorname{Lim}\left\{\left(Y_{n}, a\right), p_{n}^{n^{\prime}}, N\right\}$, where for every $n$ a pointed continuum $\left(Y_{n}, a\right)$ is a finite subbouquet of $(X, a), Y_{n} \subset Y_{n+1} \subset X$ and $p_{n}^{n+1}$ is a retraction.

(5.4) Corollary. If $(X, a)$ is a bouquet and each of its leaves is an ANR-set, then there is an inverse sequence $(\underline{Y, a})=\left\{\left(Y_{n}, a\right), p_{n}^{n^{\prime}}, N\right\}$ of finite subbouquets of $(X, a)$ such that $Y_{n} \subset Y_{n+1}$ for every $n$, all maps $p_{n}^{n^{\prime}}$ are retractions and $(X, a)$ is homeomorphic to $(\operatorname{Lim} \underline{Y, a})$.

\section{Spherical bouquets.}

(6.1) Definition. A bouquet $(X, a)$ is said to be spherical if for every leaf $X_{\lambda}$ of $(X, a)$ there is an $n_{\lambda}$ such that $X_{\lambda}$ is homeomorphic to a subset $S^{n_{\lambda}}=\left\{x \in E^{n_{\lambda}+1}\right.$ : $|x|=1\}$ of the Euclidean space $E^{n_{\lambda}+1}$.

Corollary (4.5) implies

(6.2) THEOREM. Eyery spherical bouquet is a movable pointed continuum.

(6.3) Definition. Let $(X, a)$ be a spherical bouquet and $\mathscr{X}=\left\{X_{\lambda}\right\}_{\lambda \in A}$ the family of leaves. The sequence $\underline{n}=\left(n_{1}, n_{2}, \ldots\right)$ (it may be finite) of all dimensions of leaves of $(X, a)$ is said to be the type of $(X, a)$.

By the character of $(X, a)$ we understand the sequence $\mathfrak{M}=\left(\mathfrak{M}_{1}, \mathfrak{M}_{2}, \ldots\right)$ of cardinal numbers such that for every $i$ there exist in $\mathscr{X}$ exactly $\mathfrak{M}_{i} n_{i}$-dimensionals leaves.

(6.4) Definition. If all leaves of the bouquet $(X, a)$ are $n$-spheres, then $(X, a)$ is said to be a usual n-dimensional spherical bouquet.

Theorem (3.5) and (3.7) imply

(6.5) THEOREM. Two locally connected spherical bouquets are homeomorphic if and only if they have the same type and character.

(6.6) THEOREM. If $(X, a)$ and $(Y, b)$ are locally connected spherical bouquets, then $\operatorname{Sh}(X, a)=\operatorname{Sh}(Y, b)$ if and only if $(X, a)$ and $(Y, b)$ are homeomorphic.
Proof. If $(X, a)$ and $(Y, b)$ are homeomorphic, then $\operatorname{Sh}(X, a)=\operatorname{Sh}(Y, b)$. If $\operatorname{Sh}(X, a)=\operatorname{Sh}(Y, b)$, then $X$ and $Y$ have the same Betti numbers and then they have the same type and character. It follows from Theorem $(6.5)$ that $(X, a)$ and $(Y, b)$ are homeomorphic.

(6.7) THEOREM. Every spherical bouquet has the shape of some locally connected spherical bouquet.

Proof. Let $(X, a)$ be a spherical bouquet and $\mathscr{X}$ the family of its leaves.

If $\mathscr{X}$ is finite, then $X$ is locally connected.

Let us assume that $\mathscr{X}$ is infinite. There exists, by Corollary (5.4), an inverse sequence $(\underline{X, a})=\left\{\left(A_{n}, a\right), p_{n}^{n^{\prime}}, N\right\}$ of finite subbouquets of $(X, a)$ such that $A_{n} \subset A_{n+1}$ for every $n \in N$ and all the maps $p_{n}^{n^{\prime}}$ are retractions.

It is easy to observe that we can assume that $A_{n} \neq A_{n+1}$ and $A_{n}=S_{1} \cup \ldots$ $\ldots \cup S_{n}$ for $n=1,2, \ldots$, where $S_{i} \in \mathscr{X}, i=1,2, \ldots$

We define the inverse sequence $(\underline{Y, a})=\left\{\left(Y_{n}, a\right), q_{n}^{n^{\prime}}, N\right\}$ by the formulas

$$
\begin{gathered}
Y_{n}=A_{n}, \\
q_{n}^{n+1}(x)=\left\{\begin{array}{lll}
x & \text { if } & x \in Y_{n}, \\
a & \text { if } & x \in\left(Y_{n+1}-Y_{n}\right) \cup(a),
\end{array}\right.
\end{gathered}
$$

and $q_{n}^{n^{\prime}}=q_{n}^{n+1} \ldots q_{n^{\prime}-1}^{n^{\prime}}$ for $n^{\prime}>n$.

It follows from (3.8) that the pointed continuum $(Y, \hat{a})=\operatorname{Lim}(\underline{Y, a})$ is a locally connected spherical bouquet.

We shall prove that $\operatorname{Sh}(X, a)=\operatorname{Sh}(Y, \hat{a})$. For this purpose we shall define sequences of continuous maps

$$
\text { - }
$$

$$
\begin{aligned}
& f_{n}:\left(S_{1} \cup \ldots \cup S_{n}, a\right) \rightarrow\left(S_{1} \cup \ldots \cup S_{n}, a\right), \\
& g_{n}:\left(S_{1} \cup \ldots \cup S_{n}, a\right) \rightarrow\left(S_{1} \cup \ldots \cup S_{n}, a\right)
\end{aligned}
$$

satisfying the conditions

$$
\begin{aligned}
& (1)_{n} q_{n}^{n+1} f_{n+1} \simeq f_{n} p_{n}^{n+1} \text { in }\left(S_{1} \cup \ldots \cup S_{n}, a\right), \\
& (2)_{n} p_{n}^{n+1} g_{n+1} \simeq g_{n} q_{n}^{n+1} \text { in }\left(S_{1} \cup \ldots \cup S_{n}, a\right), \\
& (3)_{n} g_{n} f_{n} \simeq \operatorname{id}_{\left(S_{1} \cup \ldots \cup S_{n}, a\right)} \text { in }\left(S_{1} \cup \ldots \cup S_{n}, a\right), \\
& (4)_{n} f_{n} g_{n} \simeq \operatorname{id}_{\left(S_{1} \cup \ldots \cup S_{n}, a\right)} \text { in }\left(S_{1} \cup \ldots \cup S_{n}, a\right) . \\
& \text { We define } f_{1}(x)=x \text { for every } x \in S_{1} .
\end{aligned}
$$

Let us assume that we have defined maps $f_{j}:\left(S_{1} \cup \ldots \cup S_{j}, a\right) \rightarrow\left(S_{1} \cup \ldots \cup S_{j}, a\right)$ satisfying the conditions $(1)_{j}$, for $j=1, \ldots, n-1$. Let us denote by $i_{n}:\left(S_{n}, a\right)$ $\rightarrow\left(S_{1} \cup \ldots \cup S_{n}, a\right)$ ard by $i:\left(S_{1} \cup \ldots \cup S_{n-1}, a\right) \rightarrow\left(S_{1} \cup \ldots \cup S_{n}, a\right)$ the inclusions, and by $\alpha:\left(S_{n}, a\right) \rightarrow\left(S_{1} \cup \ldots \cup S_{n}, a\right)$ a continuous map such that if we denote by $[\alpha] \in \pi_{l}\left(S_{1} \cup \ldots \cup S_{n}, a\right)$, where $\operatorname{dim} S_{n}=l$, the element of the homotopy group generated by $\alpha$, then $[\alpha]=\left[i f_{n-1} p_{n-1}^{n} i_{n}\right] \circ\left[i_{n}\right]$.

We define

$$
f_{n}(x)=\left\{\begin{array}{lll}
f_{n-1}(x) & \text { if } & x \in S_{1} \cup \ldots \cup S_{n-1} \\
\alpha(x) & \text { if } & x \in S_{n}
\end{array}\right.
$$


Since

$\left[q_{n-1}^{n} f_{n} i_{n}\right]=\left[q_{n-1}^{n} \alpha\right]=\left[q_{n-1}^{n} i f_{n-1} p_{n-1}^{n} i_{n}\right] \circ\left[q_{n-1}^{n} i_{n}\right]=\left[f_{n-1} p_{n-1}^{n} i_{n}\right]$,

there is a homotopy $\gamma_{n}: S_{n} \times\langle 0,1\rangle \rightarrow\left(S_{1} \cup \ldots \cup S_{n-1}\right)$ joining the maps $\left.f_{n-1} p_{n-1}^{n}\right|_{\left(S_{n, a}\right)}$ and $\left.q_{n-1}^{n} f_{n}\right|_{\left(S_{n}, a\right)}$ in $\left(S_{1} \cup \ldots \cup S_{n-1}, a\right)$. formula

The homotopy $\varphi_{n}:\left(S_{1} \cup \ldots \cup S_{n}\right) \times\langle 0,1\rangle \rightarrow S_{1} \cup \ldots \cup S_{n-1}$ defined by the $\varphi_{n}(x, t)= \begin{cases}f_{n-1}(x) & \text { for every } x \in S_{1} \cup \ldots \cup S_{n-1}, 0 \leqslant t \leqslant 1, \\ \gamma_{n}(x, t) & \text { for every } x \in S_{n} \text { and } 0 \leqslant t \leqslant 1\end{cases}$

joins the maps $f_{n-1} p_{n-1}^{n}$ and $q_{n-1}^{n} f_{n}$ in $\left(S_{1} \cup \ldots \cup S_{n-1}, a\right)$.

Let $g_{1}(x)=x$ for every $x \in S_{1}$.

It is clear that $f_{1} g_{1}(x)=x$ and $g_{1}(x)=x$ for every $x \in S_{1}$.

We define the maps $\lambda_{1}, \mu_{1}: S_{1} \times\langle 0,1\rangle \rightarrow S_{1}$ by the formulas

$$
\lambda_{1}(x, t)=\mu_{1}(x, t)=x \quad \text { for every } x \in S_{1} \text { and } 0 \leqslant t \leqslant 1 .
$$

Let us assume that the following continuous maps are defined:

$$
\begin{gathered}
g_{j}:\left(S_{1} \cup \ldots \cup S_{j}, a\right) \rightarrow\left(S_{1} \cup \ldots \cup S_{j}, a\right), \\
\lambda_{j}, \mu_{j}:\left(S_{1} \cup \ldots \cup S_{j}\right) \times\langle 0,1\rangle \rightarrow S_{1} \cup \ldots \cup S_{j}
\end{gathered}
$$

or $j=1, \ldots, n-1$, satisfying the conditions $\lambda_{j}(x, 0)=f_{j} g_{j}(x), \quad \lambda_{j}(x, 1)=x$, $\mu_{j}(x, 0)=g_{j} f_{j}(x), \mu_{j}(x, 1)=x$ for every $x \in S_{1} \cup \ldots \cup S_{j}$ and $\lambda_{j}(a, t)=\mu_{j}(a, t)$ $=a$ for every $0 \leqslant t \leqslant 1$.

Let $\beta:\left(S_{n}, a\right) \rightarrow\left(S_{1} \cup \ldots \cup S_{n}, a\right)$ be a continuous map such that .

$$
[\beta]=\left[i p_{n-1}^{n} i_{n}\right]^{-1} \circ\left[i_{n}\right] \text {. }
$$

We define the map $g_{n}:\left(S_{1} \cup \ldots \cup S_{n}, a\right) \rightarrow\left(S_{1} \cup \ldots \cup S_{n}, a\right)$ by the formula

$$
g_{n}(x)=\left\{\begin{array}{lll}
g_{n-1}(x) & \text { if } & x \in S_{1} \cup \ldots \cup S_{n-1}, \\
\beta(x) & \text { if } & x \in S_{n} .
\end{array}\right.
$$

It is easy to verify that $\left[p_{n-1}^{n} g_{n} i_{n}\right]=\left[g_{n-1} q_{n-1}^{n} i_{n}\right]$. Then there is a homotopy $\hat{P}_{n}: S_{n} \times\langle 0,1\rangle \rightarrow S_{1} \cup \ldots \cup S_{n-1}$ joining the maps $\left.p_{n-1}^{n} g_{n}\right|_{\left(S_{n}, a\right)}$ and $\left.g_{n-1} q_{n-1}^{n}\right|_{\left(S_{n}, a\right)}$
in $\left(S_{1} \cup \ldots \cup S_{n-1}, a\right)$.

The map $\psi_{n}:\left(S_{1} \cup \ldots \cup S_{n}\right) \times\langle 0,1\rangle \rightarrow S_{1} \cup \ldots \cup S_{n-1}$ defined by the formula

$$
\psi_{n}(x, t)=\left\{\begin{array}{lll}
g_{n-1}(x) & \text { if } \quad x \in S_{1} \cup \ldots \cup S_{n-1}, 0 \leqslant t \leqslant 1 \\
\hat{\gamma}_{n}(x, t) & \text { if } \quad x \in S_{n} \text { and } 0 \leqslant t \leqslant 1
\end{array}\right.
$$

is a homotopy joining the maps $p_{n-1}^{n} g_{n}$ and $g_{n-1} q_{n-1}^{n}$ in $\left(S_{1} \cup \ldots \cup S_{n-1}, a\right)$.

Since $\left[f_{n} g_{n} i_{n}\right]=\left[i_{n}\right]$ and $\left[g_{n} f_{n} i_{n}\right]=\left[i_{n}\right]$, there exist homotopies $\hat{\lambda}_{n}: S_{n} \times\langle 0,1\rangle$ $\rightarrow S_{1} \cup \ldots \cup S_{n}$ and $\hat{\beta}_{n}: S_{n} \times\langle 0,1\rangle \rightarrow S_{1} \cup \ldots \cup S_{n}$ such that $\hat{\lambda}_{n}(x, 0)=f_{n} g_{n}(x)$, $\hat{\mu}_{n}(x, 0)=g_{n} f_{n}(x), \hat{\lambda}_{n}(x, 1)=x, \hat{\mu}_{n}(x, 1)=x$ for every $x \in S_{n}$ and $\hat{\lambda}_{n}(a, t)=\hat{\mu}_{n}(a, t)$
The maps $\lambda_{n}, \mu_{n}:\left(S_{1} \cup \ldots \cup S_{n}\right) \times\langle 0,1\rangle \rightarrow S_{1} \cup \ldots \cup S_{n}$ we define by the formulas

$$
\begin{aligned}
& \lambda_{n}(x, t)=\left\{\begin{array}{lll}
\lambda_{n-1}(x, t) & \text { if } & x \in S_{1} \cup \ldots \cup S_{n-1}, 0 \leqslant t \leqslant 1, \\
\hat{\lambda}_{n}(x, t) & \text { if } & x \in S_{n} \text { and } 0 \leqslant t \leqslant 1,
\end{array}\right. \\
& \mu_{n}(x, t)=\left\{\begin{array}{lll}
\mu_{n-1}(x, t) & \text { if } & x \in S_{1} \cup \ldots \cup S_{n-1}, 0 \leqslant t \leqslant 1, \\
\hat{\beta}_{n}(x, t) & \text { if } & x \in S_{n} \text { and } 0 \leqslant t \leqslant 1 .
\end{array}\right.
\end{aligned}
$$

It is easy to verify that $\lambda_{n}$ and $\mu_{n}$ satisfy the required conditions.

It follows from conditions $(1)_{n}-(4)_{n}$, for $n=1,2, \ldots$, that $f=\left(\left(f_{n}\right)\right.$, id $\left._{N}\right)$ and $\underline{g}=\left(\left(g_{n}\right), \mathrm{id}_{N}\right)$ are maps of inverse sequences ([9], p. 41-42) and the conditions $\underline{f g} \simeq \underline{\mathrm{id}}_{(Y, a)}$ and $\underline{g f} \simeq \underline{\mathrm{id}}(X, a)$ are satisfied.

Then $\operatorname{Sh}(X, a)=\overline{\operatorname{Sh}}(Y, \hat{a})$ ([10], p. 62 and [9], pp. 41-44).

7. Fundamental sequences of usual $n$-dimensional spherical bouquets, with $n>1$. In this part we shall classify the fundamental sequences from usual $n$-dimensional spherical bouquets to usual $n$-dimensional spherical bouquets, where $n>1$.

(7.1) THEOREM. If $(X, a)$ and $(Y, b)$ are usual finite $n$-dimensional spherical bouquets, $n>1$, and $f, g:(X, a) \rightarrow(Y, b)$ are continuous maps, then $f$ and $g$ are homotopic if and only if they induce the same homomorphism $f_{*}=g_{*}: H_{n}(X) \rightarrow H_{n}(X)$ of homology groups with integer coefficients.

Proof. If $f \simeq g$, then $f_{*}=g_{*}$.

Let us assume that $f_{*}=g_{*}$.

Let $\left\{S_{1}, \ldots, S_{k}\right\}$ and $\left\{P_{1}, \ldots, P_{m}\right\}$ be the families of leaves of $(X, a)$ and $(Y, b)$, respectively, and let $\alpha_{i}:\left(S_{i}, a\right) \rightarrow\left(S_{1} \cup \ldots \cup S_{k}, a\right)$ be the inclusions.

The homomorphism $\varphi_{i}: \pi_{n}(Y, b) \rightarrow H_{n}(Y)$ defined by the formula

$$
\varphi_{i}([\beta])=\beta_{*}\left(e_{i}\right) \quad \text { for every }[\beta] \in \pi_{n}(Y, b),
$$

where $e_{i}$ is the generator of the group $H_{n}\left(S_{i}\right)$, is an isomorphism for every $i$ ([7], p. 208).

Since

$$
\varphi_{i}\left(\left[f \alpha_{i}\right]\right)=f_{*}\left(\alpha_{i}\right)_{*}\left(e_{i}\right)=g_{*}\left(\alpha_{i}\right)_{*}\left(e_{i}\right)=\varphi_{i}\left(\left[g \alpha_{i}\right]\right),
$$

for every $i=1, \ldots, k$ there is a homotopy

$$
\chi_{i}: S_{i} \times\langle 0,1\rangle \rightarrow Y
$$

joining the maps $f \alpha_{i}$ and $g \alpha_{i}$ in $(Y, b)$.

The map $\chi: X \times\langle 0,1\rangle \rightarrow Y$ defined by the formula

$$
\lambda(x, t)=\lambda_{i}(x, t) \quad \text { for every } x \in S_{i}, 0 \leqslant t \leqslant 1, i=1, \ldots, k
$$

is a homotopy joining the maps $f$ and $g$ in $(Y, b)$.

(7.2) THEOREM. Let $(X, a)$ and $(Y, b)$ be two usual l-dimensional spherical bouquets $l>1$. The fundamental sequences

$$
f=\left\{f_{n},(X, a),(Y, b\} \quad \text { and } \quad g=\left\{g_{n},(X, a),(Y, b)\right\}\right.
$$


are homotopic if and only if they induce the same homomorphism $f_{*}=g_{*}: H_{l}(X)$ $\rightarrow H_{l}(Y)$ of Vietoris homology groups with integer coefficients.

Proof. It is known ([1], p. 242) that if $f \simeq g$ then $\underline{f}_{*}=g_{*}$.

Let us assume that $\underline{f}_{*}=g_{*}$. We shall show that $\underline{f} \simeq \underline{g}$.

Let $U$ be any fixed neighborhood of $Y$ in the Hilbert cube $Q$. There exists an $\varepsilon>0$ such that $K(Y, \varepsilon) \subset U$ and we can find, by (4.3), a finite sequence $Y_{1}, \ldots, Y_{k}$ of leaves of $(Y, b)$ and a retraction $\hat{\imath}: Y \rightarrow Y_{1} \cup \ldots \cup Y_{k}$ satisfying the condition: $\varrho(\hat{r}(y), y)<\varepsilon$ for every $y \in Y$.

There are: a closed neighborhood $W$ of $Y$ in $Q$ and a continuous extension $r: W \rightarrow Y_{1} \cup \ldots \cup Y_{k}$ of $\hat{r}$ such that $\varrho(r(z), z)<\varepsilon$ for every $z \in W$.

Let $V_{0}$ be a neighborhood of $X$ in $Q$ and $n_{1}$ a natural number such that $f_{n}(V) \subset W$ and $g_{n}(V) \subset W$ for every $n \geqslant n_{1}$.

There is a $\delta>0$ such that $K(X, \delta) \subset V$. We can find, by (4.3), a finite sequence $X_{1}, \ldots, X_{m}$ of leaves of $(X, a)$ and a retraction $\hat{p}: X \rightarrow X_{1} \cup \ldots \cup X_{m}$ such that $\varrho(\hat{p}(x), x)<\delta$ for every $x \in X$.

Let $V_{0}$ be a neighborhood of $X$ in $Q$ such that there is a continuous extension $p: V_{0} \rightarrow X_{1} \cup \ldots \cup X_{m}$ of $\hat{p}$ satisfying the condition: $\varrho(p(x), x)<\delta$ for every $x \in V_{0}$. It is clear that and

$$
p \simeq \operatorname{id}_{\left(V_{0, a)}\right.} \quad \text { in }(Y, a)
$$

$$
r \simeq \operatorname{id}_{(W, b)} \text { in }(U, b) .
$$

Let $r_{1}: Q \rightarrow Q$ be a continuous extension of $r$ and $r_{n}=r_{1}$ for $n=1,2, \ldots$ The sequence $\underline{r}=\left\{r_{n},(Y, b),\left(Y_{1} \cup \ldots \cup Y_{k}, b\right)\right\}$ is a fundamental sequence. Let $i=\left\{i_{n},\left(X_{1} \cup \ldots \cup X_{m}, a\right),(X, a)\right\}$ be a fundamental sequence such that $i_{n}(x)=x$ for every $x \in Q$ and $n=1,2, \ldots$

Since $\underline{f}_{*}=\underline{g}_{*}$, then $(r f i)_{*}=(r g i)_{*}$.

The set $Y_{1} \cup \ldots \cup Y_{k}$ is an ANR-set, thus the fundamental sequences $r f i$ and $r g i$ are generated by maps ([1], p. 228). Let us denote them by $f:\left(X_{1} \cup \ldots \cup X_{m}, a\right)$ $\rightarrow\left(Y_{1} \cup \ldots \cup Y_{k}, b\right)$ and $g:\left(X_{1} \cup \ldots \cup X_{m}, a\right) \rightarrow\left(Y_{1} \cup \ldots \cup Y_{k}, b\right)$, respectively.

Since $f_{*}=(r f i)_{*}=(r g i)_{*}=g_{*}([1]$, p. 242) it follows from Theorem (7.2) that $f \simeq g$ and then $r f i \simeq r g i$.

Thus there is an $n_{0} \geqslant n_{1}$ such that

(3)

$$
\left.\left.r_{1} f_{n}\right|_{\left(X_{1} \cup \ldots \cup X_{m, a}\right)} \simeq r_{1} g_{n}\right|_{\left(X_{1} \cup \ldots \cup X_{m}, a\right)} \quad \text { in }\left(Y_{1} \cup \ldots \cup Y_{k}, b\right)
$$

for every $n \geqslant n_{0}$.

Let $n \geqslant n_{0}$, be any positive integer.

Since relations (1)-(3) are satisfied, we have

and

$$
\left.f_{n}\right|_{\left(V_{0, a)}\right.}=\left.\left.f_{n} \mathrm{id}_{\left(V_{0, a}\right)} \simeq f_{n} p\right|_{\left(V_{0, a}\right)} \simeq r_{1} f_{n} p\right|_{\left(V_{0, a}\right)} \quad \text { in }(U, b)
$$

$$
\left.g_{n}\right|_{\left(V_{0, a)}\right.}=\left.\left.g_{n} \mathrm{id}_{\left(V_{0, a}\right)} \simeq g_{n} p\right|_{\left(V_{0, n)}\right.} \simeq r_{1} g_{n} p\right|_{\left(V_{0, a}\right)} \quad \text { in }(U, b) .
$$

These relations and the relation

$$
\left.\left.r_{1} f_{n} p\right|_{\left(V_{0}, a\right)} \simeq r_{1} g_{n} p\right|_{\left(V_{0}, a\right)} \quad \text { in }(U, b)
$$

give the required relation

This proves that $\underline{f} \simeq \underline{g}$.

$$
\left.\left.f_{n}\right|_{\left(V_{0, a}\right)} \simeq g_{n}\right|_{\left(V_{0}, a\right)} \text { in }(U, b) .
$$

\section{References} [1] K. Borsuk, Concerning homotopy properties of compacta, Fund. Math. 62 (1968),
pp. 224-254. [2] - Fundamental retracts and extensions of fundamental sequences, Fund. Math. 64 (1969),

[3] - On movable compacta, Fund. Math. 66 (1969), pp. 137-146.

[4] - Some remarks concerning the shape of pointed compacta, Fund. Math. 67 (1970), pp. $221-240$.

[5] - A note on the theory of shape of compacta, Fund. Math. 67 (1970), pp. 265-278.

[6] - Theory of Shape, Warszawa 1975.

[7] Hu Sze-Tsen, Tieorija Gomotopij, Moscow 1964.

[8] W. Hurewicz and H. Wallman, Dimension Theory, Princeton 1948.

[9] S. Mardešić and J. Segal, Shapes of compacta and ANR-systems, Fund. Math. 72 (1971), pp. $41-59$.

[10] - Equivalence of the Borsuk and the ANR-system approach to shapes, Fund. Math. 72 (1971),
pp. 62-68.

[11] - Movable compacta and ANR-systems, Bull. Acad. Polon. Sci. 18 (1970), pp. 649-654.

\section{Accepté par la Rédaction le 4.11. 1974}

